\title{
Nanoscale investigations of synthetic spider silk fibers modified by physical and chemical processes
}

\author{
Gabriela M Menezes ${ }^{1}$, Florence Teulé ${ }^{2}$, Randolph V Lewis ${ }^{2}$, Luciano P Silva ${ }^{1}$ and Elibio L Rech ${ }^{1}$
}

Spider silk has biocompatibility and biodegradability properties and is known for the mechanical, physical and chemical properties that make it a promising building block in the development of novel biofibers. Its unique properties partially result from the repetitive polypeptide sequences that compose the silk proteins. The strength is related to the polyalanine motifs organized into $\beta$-sheet structures, and the elasticity is attributed to glycine-rich regions, $\beta$ turns and $3_{10}$ helix structures. Some alcohols were shown to induce $\beta$-sheet formation in spidroins and spider silk films, while water increases the overall structure ordering of silkworm fibroins. Furthermore, fiber stretching induces $\beta$-sheet formation in synthetic spider fibers. However, there is a lack of information relating the physical and mechanical behaviors that might contribute to improving the microstructure and performance of synthetic fibers. In this work, we reported the surface nanostructure and the nanomechanical behavior of synthetic spider fibers, which were composed of modified recombinant proteins to combine strength and extensibility motifs. Our present study evaluated synthetic fibers qualitatively and quantitatively, and indicated that atomic force microscopy (AFM) and scanning electron microscopy (SEM) were complementary tools to describe particular details of the surface structure and the mechanical features of synthetic spider fibers. Therefore, AFM and SEM would support the development of spinning systems and the characterization of novel biomaterials.

Polymer Journal (2013) 45, 997-1006; doi:10.1038/pj.2013.21; published online 6 March 2013

Keywords: atomic force microscopy; mechanical properties; scanning electron microscopy; spider; surface analysis; synthetic fiber

\section{INTRODUCTION}

Spiders can produce up to six different types of silk, ${ }^{1}$ which are well known for their unique mechanical properties and have been studied extensively over the years. ${ }^{2,3}$ These properties are believed to result at least partially from the repetitive polypeptide sequences that compose the silk proteins. Each type of silk is formed by conserved protein motifs that can be divided into four main groups, which confer specific characteristics (strength and elasticity) to each type of silk according to the number of repetitions and their amino-acid composition. The strength is thought to be related to polyalanine motifs organized in $\beta$-sheet structures, ${ }^{4,5}$ while the elasticity is often attributed to glycine-rich regions, ${ }^{6} \beta$ turns ${ }^{7,8}$ and semi-extended $3_{10}$ helix structures. ${ }^{9}$ Non-repetitive conserved polypeptide chains at the amino and the carboxyl termini regions have also been identified and suggested to have a role in protein folding and fiber polymerization. ${ }^{10-14}$

Dragline silk, which is used as the safety line and the frame thread of the spider web, is one of the toughest types of silk studied, it is as tough as polyaramid filaments and stronger by weight than steel. ${ }^{1}$ Dragline silk combines a reasonable viscoelasticity and extensibility with a tensile strength comparable to Kevlar, ${ }^{1,5,15}$ whereas most synthetic fibers exhibit either a low tensile strength and high extensibility or a high strength and considerable stiffness. ${ }^{16}$ Another type of silk, produced by some orb-weaving spiders, is known as flagelliform silk and composes the capture spiral, which is designed to dissipate the impact energy of the prey. Flagelliform silk is extremely elastic, extending up to $1000 \%,{ }^{17}$ and provides half the tensile strength of dragline silk, ${ }^{18}$ which results in remarkable toughness. Moreover, recent studies on spider silk have revealed other intrinsic features, such as biocompatible and biodegradable qualities. ${ }^{19-22}$

These characteristics make spider silk attractive to the field of biopolymers because spider silk can be a great material for various applications, ranging from textiles, composite materials and highperformance fibers to military (body armor and lightweight wear) and medical (artificial ligaments, drug-delivery coatings, sutures and functionalized fibers) applications. ${ }^{15,23}$ However, large amounts of silk must be available to develop such biomaterials, and their design and production require a deep knowledge of the material, which can only be obtained by relating the structural features to the function.

The complexity of the in vivo process is an obstacle to the understanding of the mechanisms involved in silk protein assembly and fiber spinning. Going from the genetic information to a solid silk

${ }^{1}$ Embrapa Genetic Resources and Biotechnology, Synthetic Biology and Nanobiotechnology Group, Brasília, Brazil and ${ }^{2}$ Department of Biology, Utah State University, Logan, UT, USA

Correspondence: LP Silva or EL Rech, Embrapa Genetic Resources and Biotechnology, Synthetic Biology and Nanobiotechnology Group, Parque Estação Biológica Final W5 Norte, Brasília DF 70770-917, Brazil.

E-mail: luciano.paulino@embrapa.br or elibio.rech@embrapa.br

Received 27 August 2012; revised 15 December 2012; accepted 28 December 2012; published online 6 March 2013 
thread involves difficulties in synthesizing the large and repetitive silk proteins $^{24}$ and in obtaining large amounts of highly purified proteins ${ }^{25}$ which are essential to achieve silk production ${ }^{26}$ at the industrial level. Another obstacle for synthetic silk production is the series of changes that the spider silk proteins experience in the solvent environment while passing through the spinning duct. ${ }^{27}$ These changes confer the secondary structures ${ }^{28}$ and the characteristics of the silk proteins and make it difficult to completely understand and reproduce such qualities.

Some reported effects of polar solvents on spider fibers, silkworm fibers and fibroins ${ }^{29-31}$ are the supercontraction and the conformational changes that result from the interaction between the solvent and the proteins. A transition from a random coil state to a $\beta$-sheet structure was described after a spidroin immersion in methanol, isopropanol and ethanol. ${ }^{30} \beta$-sheet formation induced by methanol treatment has also been demonstrated ${ }^{32}$ on recombinant spider silk films, and water was shown to increase the overall secondary structure ordering of silkworm fibroins. ${ }^{31}$ Such treatments could improve the structure and the performance of synthetic fibers, as synthetic fibers do not have the organization and properties of native fibers.

Therefore, a topographical, viscoelastic and mechanical analyses of synthetic fibers are fundamental for a better understanding of protein organization in the fiber and of the resulting mechanical properties following fiber formation. From the differences observed through surface characterization, the mechanisms of fiber formation can be adjusted to provide the synthetic fibers with the intended homogeneous morphological characteristics and also mechanical features.

Surface-texture evaluation is becoming increasingly important in a series of materials, and atomic force microscopy (AFM) is useful for studying biological complexes. AFM allows the investigation of surface structure, surface interactions and local mechanical properties because nano-indentation permits the efficient measurements of the bulk properties of the materials, such as hardness, elasticity and plasticity. Despite the applicability of AFM to biological materials, spider silk has rarely been investigated using this methodology. ${ }^{33,34}$

In this work, we demonstrated that AFM and scanning electron microscopy (SEM) were effective tools for analyzing synthetic spider fibers on nanometric and micrometric scales. Considering that the properties of spider fibers may be explored qualitatively and quantitatively, the establishment of these techniques as suitable methods to characterize spider fibers represents an important advance in the field of biomaterials. We analyzed 11 synthetic fibers, which were produced in a heterologous system and engineered so that the silk proteins had the strength and the elasticity motifs derived from dragline silk and flagelliform silk sequences, respectively. ${ }^{23}$ Eight of the fibers were submitted to post-spinning treatments (alcohol and water baths and elongation through tensile tests) to analyze whether there were any significant structural and/or functional differences compared with a control group. The surface topography, viscoelasticity, roughness, Young's Modulus, separation and interacting forces were analyzed using AFM and SEM image analyses and nano-indentation experiments (force-separation curves were obtained).

\section{MATERIALS AND METHODS}

\section{Synthetic fibers}

Recombinant synthetic fibers were engineered and produced as previously described. ${ }^{23}$ Bacteria were genetically engineered to produce a spider silk protein variant, which was composed of basic repeat units that combined a flagelliform elastic motif A1 (GPGGAGPGGA) 2 and a major ampullate silk strength motif S8, coding for a linker-polyalanine: (GGPSGPGSAAAAAAAA).
The final construct was engineered to form a $57.6 \mathrm{kDa}$ silk protein from 20 of those basic motifs (A1S820). The full amino-acid sequence was $\mathrm{MG}(\mathrm{H})_{10}$ SSGHIDDDDKHMLEDPP-[A1S8 ${ }_{20}$ silk repeat $]_{20}$-EISGSGC, which contained a histidine tag for purification purposes. The fibers were wet spun, extruded into a coagulation bath and recovered using forceps after protein purification and lyophilization. A total of eight fibers were processed and separated into four different groups according to the type of treatment that they received. A control group ( $\mathrm{C}$ group) of three non-processed fibers was also analyzed. All fibers were characterized by AFM and SEM.

The A1S820 fibers were soluble in water after the extrusion; thus, intermediate preparation steps were developed (unpublished data). As alcohols might be responsible for inducing the formation of $\beta$-sheet in silkworm fibroins, ${ }^{31,35}$ spider silk proteins ${ }^{30}$ and recombinant spider silk films, ${ }^{21,32}$ one can assume that by treating the synthetic fibers with alcohols, such as methanol and isopropanol, the protein organization and insolubility of the fibers will increase, resulting in a performance gain. Therefore, both alcohols were used in the intermediate procedures to overcome the initial solubility. Water can increase the secondary structure ordering in spider silk proteins $^{34}$ and induce $\beta$-sheet formation during recombinant spider silk polymerization, ${ }^{36}$ and it was used after the alcohol treatment for some of the fibers. The treated fibers were stretched through tensile $\operatorname{tests}^{23}$ to measure their overall mechanical performance.

The fiber treatment consisted of the following: (1) methanol treatment and $1.5 \times$ stretch (MA group); (2) methanol treatment, $1.5 \times$ stretch and water treatment (MB); (3) isopropanol treatment and $1.5 \times$ stretch (IA); and (4) isopropanol treatment, $1.5 \times$ stretch, water treatment and $1.5 \times$ stretch (IB) The stress experiments were performed in a MTS Synergie 100 system (MTS Systems Corporation, Eden Prairie, MN, USA).

\section{AFM surface analyses}

For the AFM analyses, the fibers were initially placed on paper after extrusion. Forceps were used to place them on AFM sample supports that were covered with double-sided adhesive tape. Image acquisition was performed under ambient conditions on an SPM-9600 instrument (Shimadzu, Kyoto, Japan). The images were acquired in the dynamic-phase (viscoelastic) mode ${ }^{37-39}$ using a $125-\mu \mathrm{m}$-length cantilever (spring constant of $\sim 42 \mathrm{~N} \mathrm{~m}^{-1}$, resonant frequency of $\sim 330 \mathrm{kHz}$ ) with conical tips (curvature radius $<10 \mathrm{~nm}$ ). The images were acquired as $512 \times 512$ lines at a scan rate of $0.5 \mathrm{~Hz}$. The processing consisted of an automatic global leveling and removal of the noisy lines. The images were displayed as two-dimensional height perspective. The adopted standard scanning area was $25 \mu \mathrm{m}^{2}$, and 10 different areas of each fiber were analyzed, which corresponded to a total scanned area of $250 \mu \mathrm{m}^{2}$ for each fiber.

The roughness analyses were extracted from the space-descriptive parameters $\mathrm{Rp}$ and $\mathrm{Rv}$ and from the $\mathrm{Ra}, \mathrm{Rq}, \mathrm{Rz}$ and Rzjis measurements, which were calculated as the typical height parameters. The arithmetic average roughness (Ra) corresponds to the absolute values of roughness measured from a mean line over the sampling length. The root-mean-square roughness (Rq) is a quadratic mean also based on the measured absolute values. The maximum height $(\mathrm{Rz})$ was calculated from the peak-to-valley height, while Rzjis corresponds to the 10-point mean roughness based on the five highest peaks and lowest valleys in the entire sampling length. In addition, the maximum peak height $(\mathrm{Rp})$ was calculated based on the distance between the average of the section curve and the highest peak encountered on the scanned surface, and the maximum valley depth ( $\mathrm{Rv})$ corresponds to the distance between the average of the section curve and the lowest valley found. $\mathrm{Rp}+$ $\mathrm{Rv}=\mathrm{Rz}$. This range of parameters was selected to include the relevant parameters that could describe and explain the investigated topographical properties of the synthetic fibers as they were spun and after the described treatments. Additionally, viscoelastic features were qualitatively investigated on the phase images.

\section{Force spectroscopy analyses}

Force spectroscopy measurements were also performed under ambient conditions on the same AFM instrument used for image acquisition. The same $\mathrm{Si}_{3} \mathrm{~N}_{4}$ cantilever with a pyramidal tip and a spring constant of $0.57 \mathrm{nN} \mathrm{nm}^{-1}$ was used. The experimental procedures were performed in 
contact mode at a constant loading rate of $1 \mathrm{~nm} \mathrm{~s}^{-1}$. The force fit range was set to auto. Fifteen indents on different areas were performed for each fiber for a total of 165 measurements, and a commercial software was used for data analyses (SPIP, Image Metrology, Kongens Lyngby, Denmark). The Young's moduli and the forces derived from the retraction curves were calculated using the analytical Sneddon model of cone indentation. ${ }^{40,41}$

In addition to topography (height images) and viscoelasticity (phase images), which were acquired through surface scanning, ${ }^{42}$ the AFM provided the force-distance curves, which represented the interaction forces between the tip and the sample vs the tip-sample distance. To obtain this representation, the tip (or the sample) was moved in a vertical direction (nano-indentation), and the deflection of the cantilever was measured. The provided information included the transverse Young's Modulus, which reflected the local elasticity of the sample; the forces acting on the probe, which comprised the attractive and the adhesive forces (snap-in and detach, respectively); and the maximum loading forces. The tip-sample separation measurement (deformation/penetration depth) also reflected the mechanical characteristics of the sample. ${ }^{42-44}$

\section{SEM analyses}

The fibers were examined using a SEM. The samples were coated $1 \times$ with gold to ensure their conductivity (Emitech K550, Emitech Ltd., Ashford, UK ) and viewed on a DSM 962 Zeiss (Carl Zeiss, Oberkochen, Germany) at a $10 \mathrm{kV}$ voltage. The fibers were imaged with various magnifications from $\times 100$ $\times 3000$. However, only the $\times 3000$ images are shown in the results because they offered a larger spectrum of details.

\section{Statistical analysis}

All data are presented here as the means \pm s.e.m., and the statistical significance was determined by calculating a probability value $(P)$ with Student's $t$-test. The differences were considered significant when $P<0.05$ or $P<0.01$. The statistical analysis was performed using the software Assistat 7.5 Beta (Assistat, Campina Grande, Brazil).

\section{RESULTS}

\section{Fiber morphology}

The AFM images showed the representative structural differences among the analyzed fibers. Even the fibers in the same group had inhomogeneous topographical characteristics, and different scanned areas of the same fiber also showed distinct characteristics. Because of the difficulties that arose during the surface scanning with AFM, a micrometric analysis was performed using SEM. The heterogeneity found in the nanometric scale was confirmed by the SEM images (Figure 1), and a large range of fiber diameters could be observed, as previously cited. $^{23}$

The AFM analyses supported this heterogeneity, which included the proportion of elastic and viscous areas and the size of the protein aggregations. The topographic information was represented in threedimensional height images (Figures 1a-e).Viscoelastic features were observed in the phase images (Figures $1 \mathrm{f}-\mathrm{j}$ ). The dark areas correspond to the viscous surfaces, while the lighter areas correspond to the elastic surfaces.

No clear pattern was found for any of the groups. All groups exhibited topographic and viscoelastic variations among the fibers. The size of the granules on the surface of the fibers varied in different areas of a single fiber, and even greater differences were found when different fibers were compared. In most cases, the phase images showed a predominance of rough areas with elastic spots or strips (Figures 1f and i).

The SEM images showed the characteristics, such as the smoothness, the shine and the presence of protuberances, of the fibers. The images revealed grainy surfaces for some of the fibers (Figures $1 \mathrm{k}-\mathrm{m}$ ). The presence of bubbles was also observed in some cases (Figure 1k).
Voids and cracks in some of the scanned areas were observed (Figure $1 \mathrm{k}$ ), and the longitudinally oriented fissures were clearly observed in the representative images (Figures 11 and $\mathrm{m}$ ).

\section{Fiber topographic measurements}

The mean values of the roughness parameters of the analyzed synthetic fibers are displayed in Figure 2. All studied groups presented a similar general pattern to the six parameters. $\mathrm{Ra}$ is one of the most common parameters used to describe roughness in surface-characterization studies. Our results showed that the treatments did not significantly alter the $\mathrm{Ra}$ compared with the $\mathrm{C}$ group (Figure 2a). However, significant differences were observed among the treated groups, as the isopropanol-treated groups showed a decrease in $\mathrm{Ra}$ compared with the methanol-treated groups $(P<0.05)$. The IA group decreased $31 \%$ in $\mathrm{Ra}$ compared with the MA group and $28 \%$ compared with the MB group $(P<0.05)$, while IB decreased 27 and $23 \%$ compared with $\mathrm{MA}$ and $\mathrm{MB}$, respectively $(P<0.05)$. No significant differences in Ra were observed within the isopropanoltreated groups or the methanol-treated groups.

Other roughness parameters may enrich the understanding of the surface features. Therefore, the values of $\mathrm{Rq}, \mathrm{Rv}, \mathrm{Rp}, \mathrm{Rz}$ and Rzjis obtained during fiber scanning were also plotted and analyzed.

Comparison of Rz, Rzjis, Rq and Rv plots to the Ra plot showed that variations in the behavior of the groups were very subtle. The $\mathrm{Rq}$ measurements showed that the groups behaved similarly to Ra results because none of the treated groups diverged statistically from the $\mathrm{C}$ group (Figure $2 \mathrm{~b}$ ). As we noticed in the $\mathrm{Ra}$ analysis, the isopropanoltreated groups were significantly different from the methanol-treated ones $(P<0.01)$, but the groups of fibers that received the same alcohol treatment did not show any significant discrepancies (Figure $2 b$ ).

The $\mathrm{Rv}$ analysis showed no significant differences among the $\mathrm{C}$ group, the MA group and the MB group (Figure 2c). Although the IB group did not differ significantly from the $C$ group and $M B$, it presented a $31 \%$ decrease compared with MA. The IA group exhibited no significant differences compared with IB but decreased by 24,38 and $30 \%$ compared with the $\mathrm{C}$ group, $\mathrm{MA}$ and $\mathrm{MB}$, respectively $(P<0.01)$.

Rp diverged substantially from the general pattern that the other analyzed AFM roughness parameters showed (Figure 2d). The maximum-peak-height analysis showed that the $\mathrm{C}$ group and the methanol-treated groups did not statistically differ from each other, and IA only differed from MA $(P<0.01)$. However, the IB group diverged substantially from the $\mathrm{C}$ group, $\mathrm{MA}$ and $\mathrm{MB}$, showing significant decreases of 34, 40 and 35\%, respectively $(P<0.01)$. The IB group also showed the lowest s.e.'s of all analyzed groups.

The $\mathrm{Rz}$ and Rzjis analyses revealed a similar pattern of the behavior of the studied groups (Figures 2e and f). The methanol-treated groups did not show any significant differences among themselves and compared with the $\mathrm{C}$ group, but the isopropanol-treated groups diverged significantly from all other groups. The $\mathrm{Rz}$ analysis showed that the IA values decreased by 21,32 and $25 \%$ compared to the $C$, MA and MB groups, respectively $(P<0.01)$. The values of the IB group also significantly decreased by 21,34 and $26 \%$ compared with the $\mathrm{C}, \mathrm{MA}$ and $\mathrm{MB}$ groups, respectively $(P<0.01)$. The Rzjis analysis also revealed a decrease in roughness after the isopropanol treatment: IA appeared 20, 32 and $24 \%$ less rough than the C, MA and MB groups, respectively. The roughness of IB also significantly decreased by 21,33 and $26 \%$ compared with the $\mathrm{C}$ group and the methanoltreated MA and MB groups, respectively $(P<0.01)$.

The obtained results led to a new set of experiments to test the mechanical properties of the analyzed fibers with a focus on the 
a

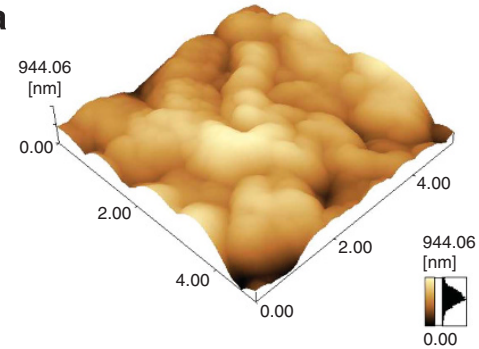

b

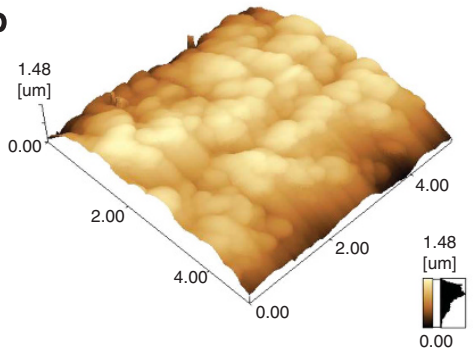

C

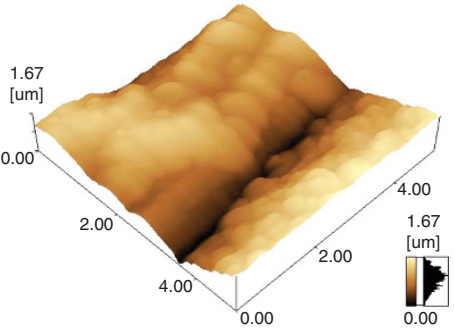

d

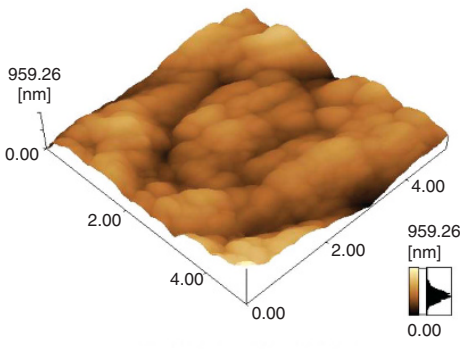

e

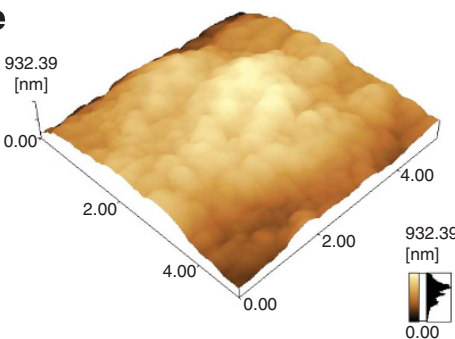

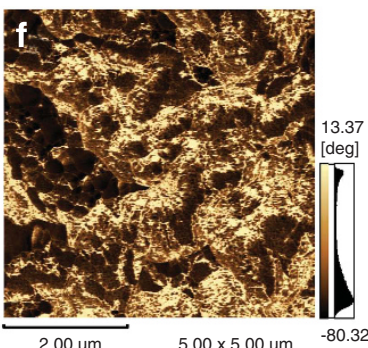
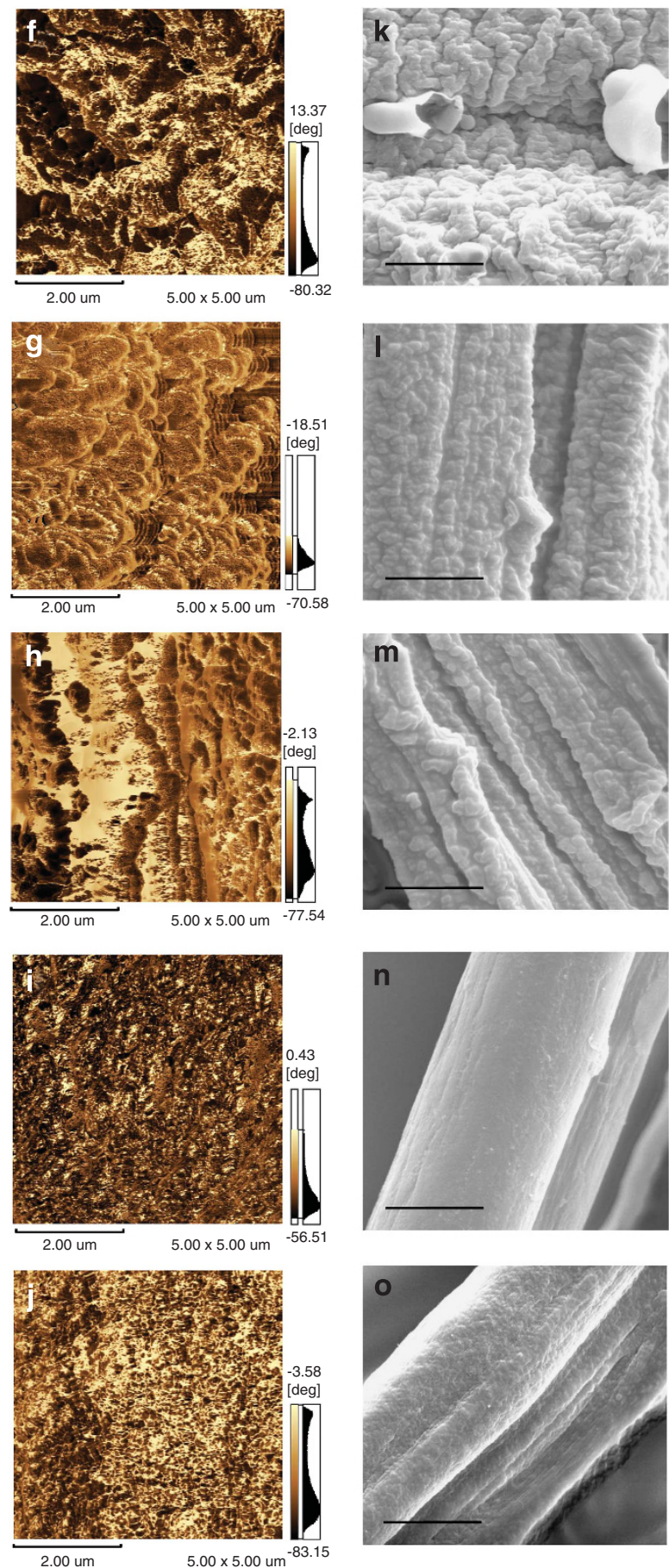
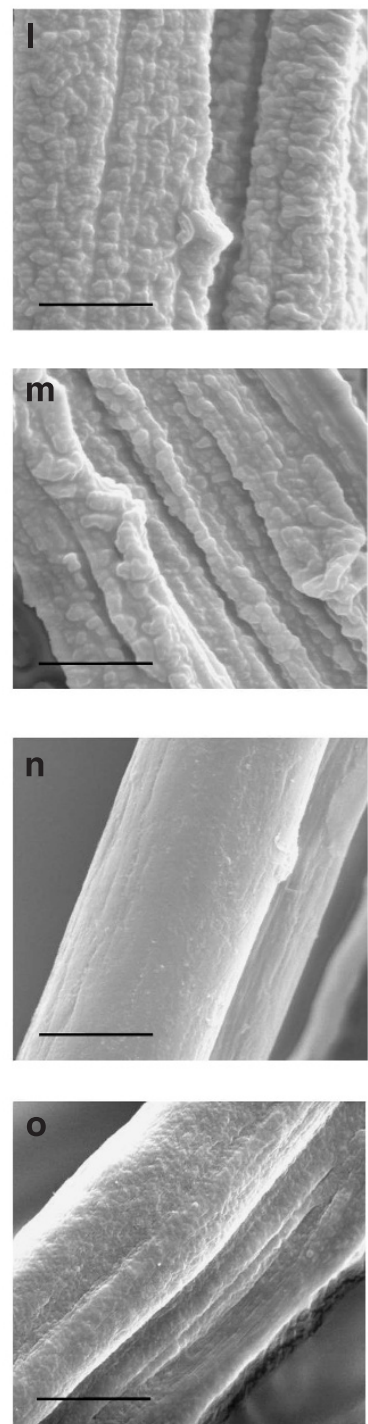

Figure $1 \mathrm{AlS}_{20}$ surface AFM and SEM images. From left to right: Column I, topographical three-dimensional height AFM images; Column II, $5 \times 5 \mu \mathrm{m}^{2}$ phase images that demonstrate the viscoelastic characteristics, the scale bar corresponds to $2 \mu \mathrm{m}$; Column III, SEM $\times 3000$ images, the scale bar corresponds to $10 \mu \mathrm{m}$. Each line represents one set of images concerning one fiber of a specific group. Panels $\mathbf{a}, \mathbf{f}$ and $\mathbf{k}: \mathrm{C}$ group; $\mathbf{b}, \mathbf{g}$ and $\mathbf{~ : ~} \mathbf{M A}$ group; c, $\mathbf{h}$ and $\mathbf{m}$ : MB group; $\mathbf{d}, \mathbf{i}$ and $\mathbf{n}$ : IA group; and $\mathbf{e}, \mathbf{j}$ and $\mathbf{o}:$ IB group.

different types of treatment that we used and their influence on the measured properties.

\section{Fiber force measurements}

Various solvent treatments, such as ethanol, methanol, water and urea, were shown to alter the mechanical properties of fibers. ${ }^{29,41} \mathrm{~A}$ common feature of spider dragline silk is its apparent ability to supercontract when submerged in a solvent such as water. ${ }^{16,29,34,43}$ Furthermore, reports on the effect of alcohol on spider proteins indicated that alcohols can induce $\beta$-sheet formation, ${ }^{30,31}$ which might enable the formation of fibers with an increased performance. Considering the possible resulting implications, it is of great importance to examine the mechanical behavior of synthetic fibers with and without the treatments described. 

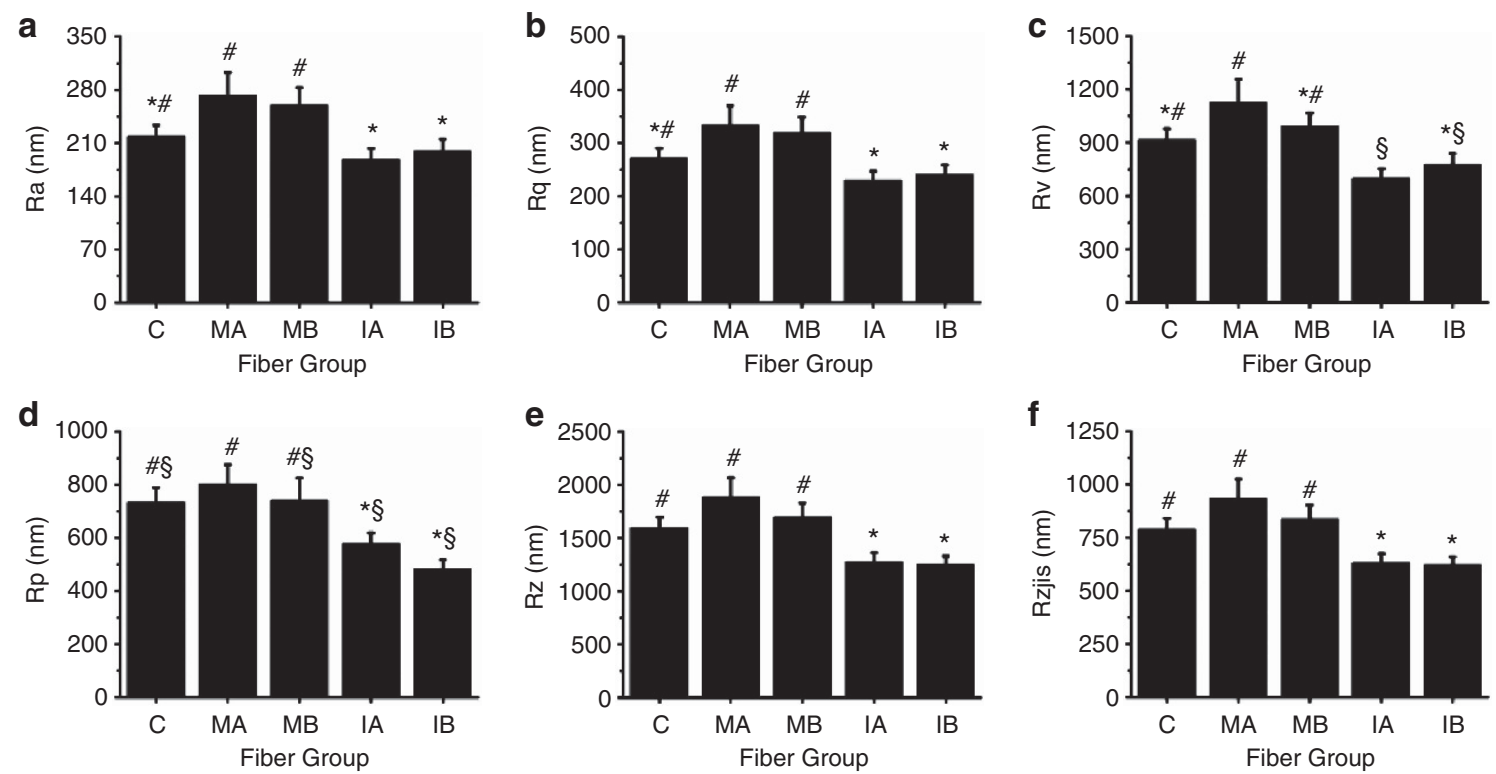

Figure 2 AFM roughness analysis of $A 1 S 8_{20}$ fibers. The analyzed parameters were arranged on the $y$ axis of each graph. Ra is the arithmetic average roughness, $R q$ is the root-mean-square roughness, $R z j i s$ is the 10-point average roughness, $R z$ is the maximum height, and $R p$ and $R v$ are the average height and the average depth. The symbols represent similar quantities among the groups.
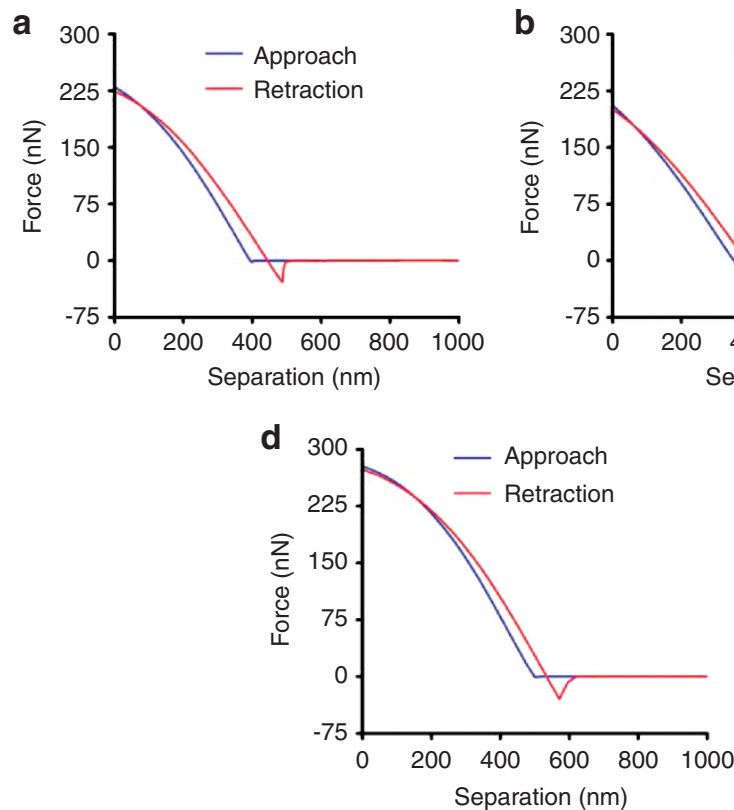
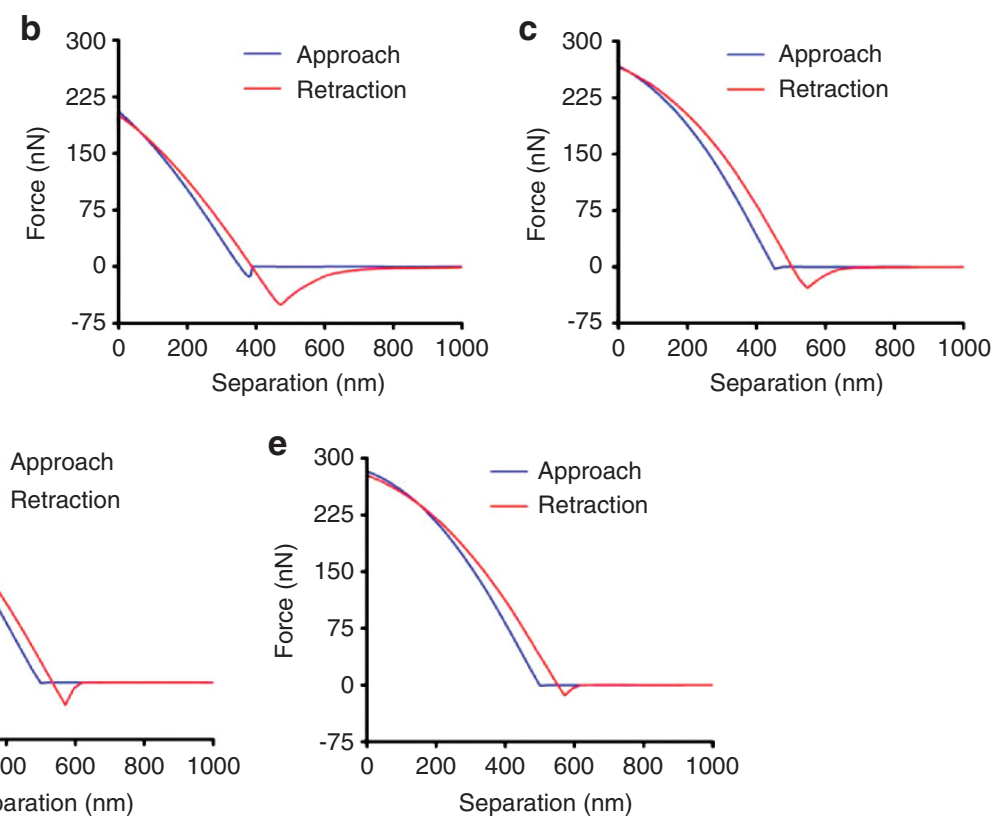

Figure 3 Force-separation curves for all five A1S8 20 groups. (a) Group C, (b) Group MA, (c) Group MB, (d) Group IA and (e) Group IB. The approach and retraction curves are shown.

The force-curve analysis provided relevant information regarding the mechanical properties of the analyzed sample. The curves shown in Figure 3 reveal substantial differences among the groups. The observed heterogeneity implied that variations existed even among the curves obtained for a single fiber. We noticed the increment in the penetration distance (separation) with increasing applied force and the discontinuity points that resulted from the interaction forces between the tip of the microscope and the surface of the fibers. The approach line and the retraction line did not coincide, which corresponded to the hysteresis of the force-separation curves and resulted from the adhesive forces that hindered the detachment of the tip. ${ }^{44}$ Hysteresis may also be an evidence of an elastic-plastic behavior because perfectly elastic materials do not experience this phenomenon. ${ }^{45}$

Young's Modulus. Stress-strain experiments were previously conducted, ${ }^{23}$ and the Young's modulus values were measured $(760 \pm 540 \mathrm{MPa})$. Tensile tests may provide important information on the mechanics of the entire sample length, but measurements on the nanometric scale might help us fully understand the features of 


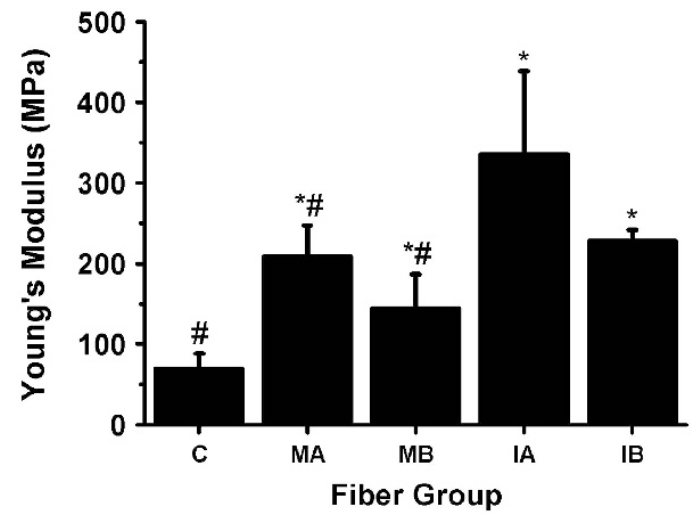

Figure 4 Average Young's modulus, which reflects the local elasticity of the synthetic spider fibers, as measured using nano-indentation. The symbols represent similar quantities among the groups.

synthetic fibers. The experiments performed in this study provided the transverse Young's modulus instead of the longitudinal elastic modulus that was measured in the tensile tests.

We used Hertzian fits to the force curves to quantitatively compare the Young's modulus for each fiber group (Figure 4). The lowest value measured corresponded to the $\mathrm{C}$ group, which differed significantly from the IA and the IB groups $(P<0.05)$. There were no significant differences between the IA and the IB groups, and the methanoltreated groups did not differ significantly among themselves or from any other groups. The IA group presented the highest mean module of all analyzed groups, showing a 4.9-fold increase compared with the C group. IB recorded the second highest value with a 4.3 -fold increase. The modulus values also varied at several different positions in each fiber, which contributed significantly to the high s.e.'s demonstrated.

Measured forces. Three types of forces were derived from the force curves generated in the indentation experiments. Such forces, associated with the elastic modulus, might give important information on the mechanical properties of the fibers. The maximum loading force, which was extracted from the approach curve, is plotted in Figure 5a. No significant difference was found between the IA and the IB groups, but they differed considerably from the $\mathrm{C}$ and $\mathrm{MB}$ groups. The isopropanol-treated groups had significantly higher values with lower s.e.'s, increasing by 2 -fold and 2.2 -fold, respectively, compared with the $\mathrm{C}$ group and increasing by 29 and 34\% compared with the MB group $(P<0.01)$. The MA values significantly increased by $75 \%$ compared with the $\mathrm{C}$ group $(P<0.01)$ but did not differ significantly from any other groups. The s.e.'s obtained for the methanol-treated groups were considerably high.

The snap-in and the detach forces are represented in Figures $5 b$ and $c$, which illustrate the forces resulting from the surface interactions of the fibers with the tip used for the nano-indentation experiments. Snap-in corresponds to the attractive forces between the sample surface and the tip during indentation, and detach force corresponds to the point where the tip and the surface lose contact, which provides information on the adhesive forces resulting from the retraction of the tip. The isopropanol-treated groups presented statistically similar snap-in and detach force values, but they differed considerably from the other groups $(P<0.01)$. IA decreased by $94 \%$ (snap-in) and $57 \%$ (detach force) compared with the $\mathrm{C}$ group, while IB decreased by $95 \%$ in the snap-in and $66 \%$ in the detach force $(P<0.01)$. The MA group
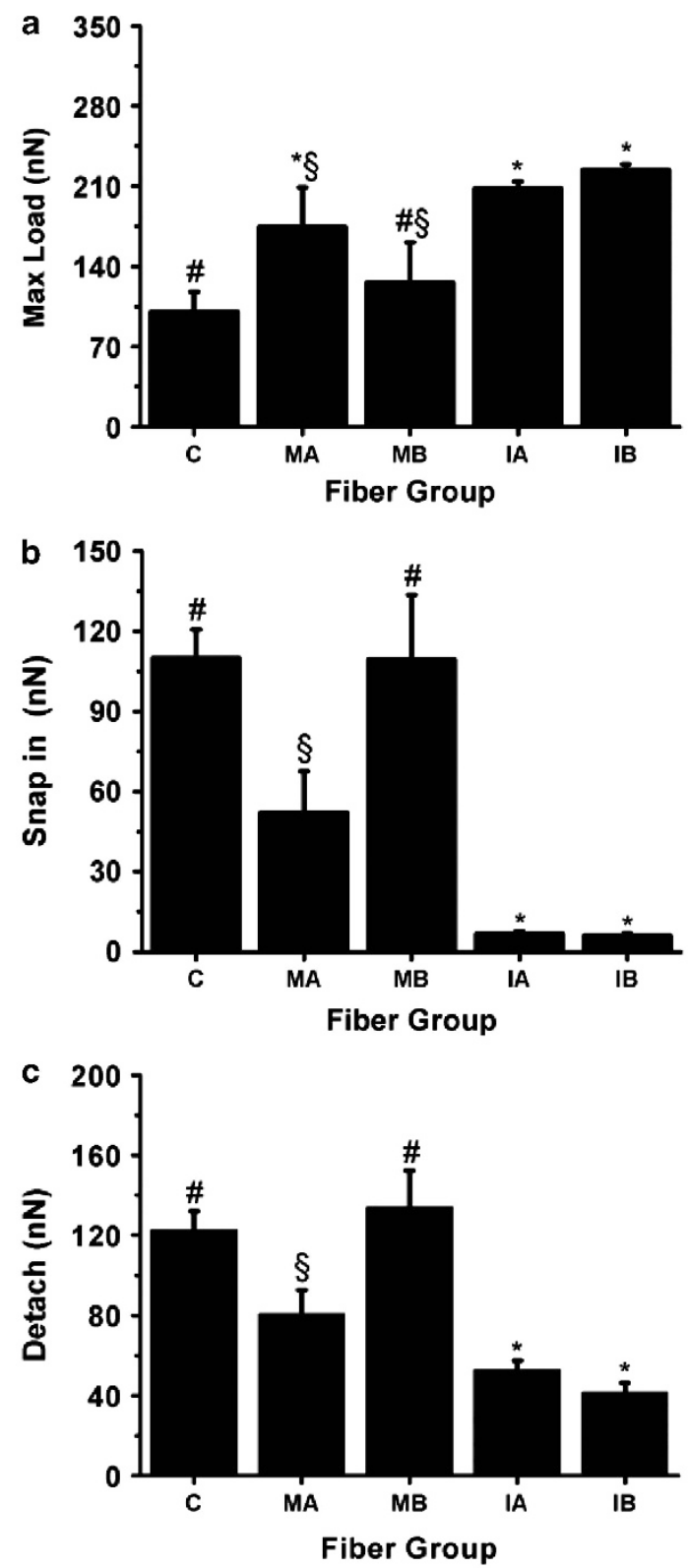

Figure 5 Measured forces that reflect the mechanical properties of the synthetic fibers. (a) Maximum loading force obtained during indentation. (b) Average snap-in force corresponding to the attractive properties of the surface. (c) Average detachment force corresponding to the adhesive forces, measured at the point where the tip loses contact with the sample. The symbols represent similar quantities among the groups.

presented significant differences from all other groups, and the measured values for snap-in and detach force were 53 and $34 \%$, respectively, lower than the values observed for the $\mathrm{C}$ group. The $\mathrm{MB}$ group showed the highest values among the treated groups $(P<0.01)$ and presented no significant differences from the $\mathrm{C}$ group.

Measured displacement. The detach separation, which is the distance between the tip apex and the maximum indentation point at detachment, is represented in Figure 6 and showed a similar pattern to the plots of the snap-in and the detach force. Very low values of detach separation were found for the IA and IB groups, which differed 


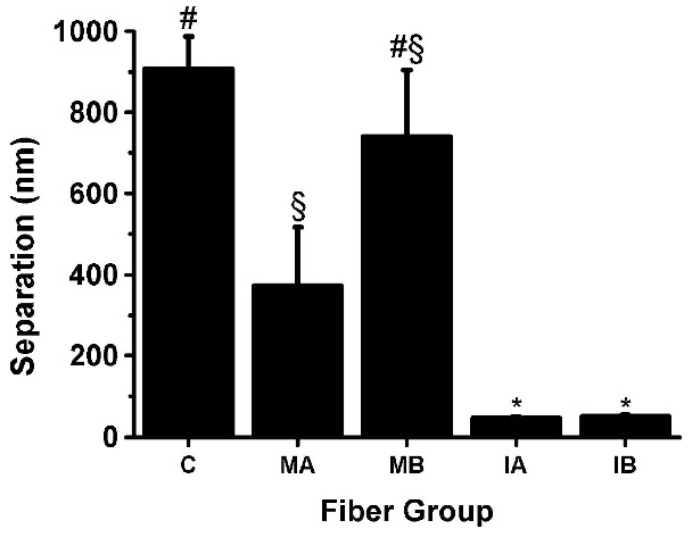

Figure 6 Tip-sample distance (maximum penetration depth) during nano-indentation. The symbols represent similar quantities among the groups.

significantly from the other groups $(P<0.01)$, and both corresponded to $5 \%$ of the values measured for the $\mathrm{C}$ group. The results showed no significant differences among the isopropanol-treated groups. The MA group decreased detach separation parameter by $60 \%$ compared with the $\mathrm{C}$ group $(P<0.01)$, but no significant differences were observed between the $\mathrm{MB}$ and the $\mathrm{C}$ group or the MA group.

\section{DISCUSSION}

We found important differences among the different treatment groups, as the fibers in both isopropanol-treated groups had smoother surfaces in most cases and a significant improvement in their mechanical behavior.

The AFM and SEM images established no clear pattern for any of the analyzed fiber groups. It was possible to verify the heterogeneity of the synthetic fibers, which included the topography, viscoelasticity, diameter and overall appearance. The observed variations are possibly related to the mechanism of protein assembly, which is not fully understood. The alanine-rich and glycine-rich motifs of native fibers were expected to fold in specific secondary structures. ${ }^{4,5,9,46}$ However, under these conditions, the synthetic proteins might not fold properly, resulting in non-uniform characteristics. Moreover, the lack of the $\mathrm{C}$ terminus and $\mathrm{N}$ terminus in synthetic spider silk proteins, which may be involved in the proper folding of proteins and the fiber polymerization, ${ }^{10-14}$ may have contributed to the heterogeneous and possibly deficient structure. The tensile tests might also have contributed to some of the irregular features observed. There is no evidence that the contaminants resulted from the process of fiber formation.

Native Araneomorphae spider silk fibers exhibit a smooth surface $^{47,48}$ (Figure 7). The fibers in the IA and IB groups exhibited the smoothest and shiniest surfaces, whereas the surfaces of the fibers in the MA, MB and the $\mathrm{C}$ groups appeared irregular. Therefore, the isopropanol treatment appeared to diminish the topographical irregularities. The alternation of smooth and grainy areas suggests that the isopropanol might have permeated the fibers and induced some reorganization that smoothened the fibers. As the IB group fibers seemed to be the smoothest of all fibers analyzed, we concluded that water might also interact with the microstructure of the fibers. Water may have allowed the reorientation of the amorphous chains, which was enhanced by fiber stretching, and caused new hydrogen bonds to form after the water evaporated, resulting in the structural changes. ${ }^{29}$

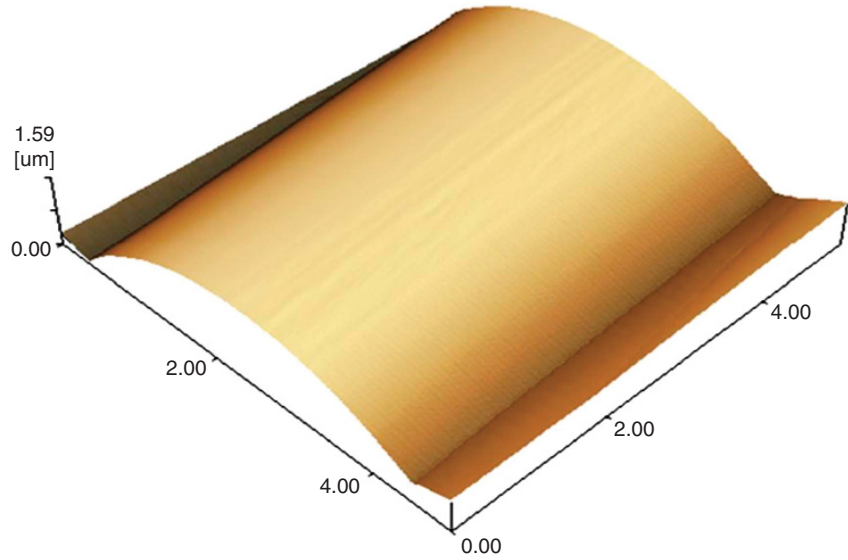

Figure 7 AFM-topographical three-dimensional height $5 \times 5 \mu \mathrm{m}^{2}$ image of a Nephila clavipes native dragline silk fiber with evident surface smoothness.

The roughness parameters that we analyzed also demonstrated the differences among the treated groups. The data obtained for the groups IA and IB showed that the isopropanol treatment reduced the roughness of the synthetic fibers even with the following stretching and posterior water treatment (IB). Such results indicated that isopropanol could promote a uniform increase through the interaction of the solvent with the protein chains. The addition of alcohol would weaken the hydrophobic interactions that stabilize the spidroins, intramolecular hydrogen bonding sites would be strengthened and the extended helices ( $3_{10}$ helices) would be stabilized. ${ }^{49}$ The conformational change of the random coil structures into $\beta$-sheets has been reported for spidroins in solution. ${ }^{30}$ Therefore, an improvement in the fiber microstructure organization may decrease the roughness of the $\mathrm{A} 1 \mathrm{~S} 8_{20}$ synthetic fiber.

The methanol-treated groups did not differ significantly from the $\mathrm{C}$ group in any of the parameters analyzed because they demonstrated a tendency towards increasing roughness. Although methanol was reported to induce the formation of $\beta$-sheets ${ }^{30,31,32,35}$ and solvents with higher polarity have a greater ability to interact with spider silk proteins, ${ }^{29}$ methanol was inefficient in smoothing the fiber surface. This result might occur because the solvent polarity, the dielectric constant and the speed with which methanol induces the conformational changes were greater than those observed for isopropanol. ${ }^{31}$ The interactions between methanol and the proteins, which were more aggressive than those derived for isopropanol, may have led to a type of reorganization that was harmful to the characteristics of the synthetic fibers. The increase in local roughness was also observed on the recombinant spider silk films after methanol treatment ${ }^{32}$ and was attributed to the structural rearrangements on the protein film surface, which was evidenced by the formation of large $\beta$-sheet nano-crystals.

Variable mechanical performances were noticed in all groups of fibers, and they considerably exceeded the variability levels exhibited by native silk fibers ${ }^{48,50}$ and synthetic polymeric fibers. ${ }^{23}$ The longitudinal Young's modulus of these fibers was measured to be $760 \pm 540 \mathrm{MPa}$, which provided important information on the overall mechanics of the fibers. ${ }^{23}$

This study also measured the transverse Young's modulus of the fibers, and the Hertzian fits of the force curves provided a quantitative comparison among the analyzed groups. The values found for different measurements of a single fiber indicated that the Young's modulus was not uniform across the surface, which is in accordance 
with the high s.e.s. The isopropanol-treated groups recorded the highest Young's modulus values, indicating that they had a higher local elasticity than the other groups.

The elasticity measurement is directly related to the local stiffness of the analyzed material, which is possibly related to their structural organization (formation of secondary structures such as the $\beta$-sheets). ${ }^{9,51}$ Higher values of the Young's modulus corresponded to greater stiffness/toughness of the analyzed area, ${ }^{51}$ which may be associated with better structuring of the surface (proper formation of the secondary structures). Moreover, the significant differences in the modulus of the crystalline and amorphous $\mathrm{YCo}_{2}$ films, which we analyzed using AFM, indicated that the crystalline phases were stiffer. $^{40}$ Thus, the low elasticity presented by the non-treated group suggests a weak organizational structure.

The self-assembly process of native proteins is initiated by the environmental changes in the spider gland ${ }^{27}$ and the applied shearing forces, which promote alignment and packaging. The hydrophobic polyalanine segments associate and are exposed to an increasing hydrophobicity, which can initiate the folding into $\beta$-sheets that result in the formation of a series of interchain hydrogen bonds. ${ }^{52}$ Without the conditions present in the lumen of the silk glands and because of the differences in the forces applied during fiber polymerization, the polyalanine segments may not organize properly, which prevents the formation of all $\beta$-sheets and hydrogen bonds that are necessary for the correct structuring of the synthetic fibers. Therefore, the synthetic fibers exhibit a reduced stiffness resulted from the low structural organization, which is reflected in the lower Young's modulus values of the fibers of the $\mathrm{C}$ group.

As previously suggested, isopropanol treatment could induce changes in the elastic behavior of the fibers, which might result from structural modifications. The fibers treated with methanol also tended to have higher elasticity than the $\mathrm{C}$ group. Previous reports indicated that these alcohols might promote the transition from a disordered state and the proper formation of secondary structures, ${ }^{30,31}$ which might explain the results obtained for the fibers studied in this work. Higher $\beta$-sheet contents are suggested to be related to the increased stiffness of the spider fibers. ${ }^{42}$ The greater structural organization of the fibers induced by the treatments would result in increased stiffness, which is directly reflected in the Young's modulus values recorded for the treated groups, in particular, IA and IB. After the polyalanine segments correctly folded, the other motifs may have also correctly folded, which resulted in an increased elasticity of the treated fibers.

However, the groups that were subsequently treated with water had a lower average modulus than those treated with only alcohol and stretched (MB compared with MA and IB compared with IA) (Figure 4). The loss of elasticity after immersion in water, which was represented by a decrease in the Young's modulus, had been described for native spider silk. ${ }^{34}$ The observed tendency may reflect the structural changes induced by the interaction with water. The silk produced by some insects and those produced by spiders can contract when they are in contact with polar solvents (water in particular), which results in a temporary loss of stiffness (supercontraction/ plasticizing effect). This effect implies substantial changes in the fiber organization and their mechanical properties. ${ }^{34,42,53}$

The differences observed among the water-treated groups and those treated only with alcohol can be explained by the described plasticization effect. Water penetrated the fiber and was incorporated into the amorphous matrix, which reduced the interaction between the polymer chains. The new hydrogen bonds formed between the water molecules and the amorphous chains, and the increased mobility of the chains promoted a temporary loss of rigidity. ${ }^{29,34}$ The loss of rigidity increased the deformation capacity, a property of plastic materials, ${ }^{45}$ which reduced the modulus. This loss of rigidity might be related to the low degree of orientation of the amorphous matrix of the fiber in a polar solvent such as water. ${ }^{43}$ Although the effect is described as temporary in most cases comprising native fibers, we suggest that the modifications might be more stable in synthetic fibers.

The supercontraction of spider fiber is related to the proportion of the $\beta$-sheets and the ability of the solvent to interact with the proteins, ${ }^{42}$ which depends on its polarity and the size of its molecules. $^{29}$ The water-treated groups had a lower modulus than those treated only with methanol or isopropanol and stretched, which could be explained by an increase in the supercontraction effects as the solvent polarity increased. This result suggests that the intramolecular and intermolecular hydrogen bonds were altered after the water treatment. ${ }^{29,42}$ Moreover, because the fibers were stretched after the methanol and isopropanol treatments, the elongation might also change the initial microstructure and molecular structure of the fibers. Possible changes in the microstructure of the high-molecular-weight poly (amino acid) and the spider fibers during the stretch experiments were reported, ${ }^{29}$ and the rearrangement of the native spider fibrils in a more packed structure when they were subjected to stress was also described. ${ }^{54}$ The subsequent treatment of the stretched fibers with water disoriented the molecular chains instead of regaining the original conformation and promoted the formation of new hydrogen bonds; ${ }^{29}$ thus, the resulted fibers were structurally different from the unprocessed ones.

The results indicated a certain degree of stability of the modifications induced by the treatments because the mechanical response of the fibers was different even after the solvents evaporated. Stretching and the subsequent treatment with water could not reverse the gain in elasticity that the isopropanol treatment induced. Thus, the changes in the secondary structure of the synthetic fibers, which were indicated by an improvement in the fibers' performance, could be induced in a more stable way. The methanol treatment could not induce any statistically significant change in the fibers' performance as previously stated. However, because the treated groups tended to increase their elasticity, stable structural changes might exist.

The forces measured in the nano-indentation experiments also revealed important features of the synthetic fibers. The maximum force that was directly applied reflects the characteristics of the analyzed material: high force values suggest hard surfaces with steep force curves, while low values refer to soft surfaces. ${ }^{41,55}$ The hardness is the maximum load divided by the contact area between the tip and the sample after the sample has recovered elastically. ${ }^{40}$

Our results indicate that the isopropanol treatment increased the hardness of the fibers, as evidenced by the higher values of maximum force measured. The methanol treatment increased the hardness (MA) but might allow at least a partial reversal of this state, as the MB group and the $\mathrm{C}$ group were not significantly different. The observed increase in hardness possibly reflects that alcohols, particularly isopropanol, induced the formation of $\beta$-sheets. ${ }^{30}$ When the proteins passed from a random coil state to a higher level of organization (proper formation of the secondary structures), this structural change was reflected in the mechanical properties. A hierarchical model of structure in the spider fibers suggests that silk polymerization relies on interdependent steps. ${ }^{56}$ As the aggregation mechanism of silk should be a nucleation-dependent process, ${ }^{57}$ we assumed that the proper formation of the $\beta$-sheets would allow other structures to arrange themselves properly. The attractive (snap-in) 
and adhesive (detach) forces, which reflect the tip-sample interactions during indentation, were measured in terms of an interaction potential. ${ }^{45}$ The interaction forces recorded for IA and IB were substantially lower than those for the other groups, which suggests that the fibers in the isopropanol groups were structurally changed possibly because of the molecular reorganization induced by the alcohol interaction.

The snap-in forces were directly measured from the cantilever deflection. The jump to contact relies on the attractive forces overcoming a minimum potential, which depends on the density of the materials, their instantaneous dipole interaction and the medium (vacuum, air and liquid). ${ }^{45}$ The materials with different structural and chemical compositions would exhibit distinct responses to the tip approximation of the AFM.

As the detach forces arise from plasticity and adhesion, ${ }^{45}$ plastic materials are more prone to high pull-off forces, as suggested in this study. Assuming that the isopropanol induced structural changes in the fibers, the above statement agrees with the results obtained because the $\beta$-sheet formation provides stiffer and more elastic fibers.

\section{CONCLUSION}

Synthetic spider fibers have particular structural and mechanical features that can be improved using an isopropanol treatment. The surface irregularity, elasticity and strength of the fibers likely depend on their molecular organization, and an alcohol bath appears to initiate the transition of random coil structures to $\beta$-sheets, which result in smoother, stronger and more elastic fibers. AFM complemented by SEM was able to qualitatively describe the synthetic fibers and quantify their roughness and mechanical properties. This method represents a significant step forward in spider silk characterization and application as a biomaterial.

\section{CONFLICT OF INTEREST}

The authors declare no conflict of interest.

\section{ACKNOWLEDGEMENTS}

We are grateful to Ana CMM Gomes from Bio Image Laboratory from Embrapa Genetic Resources and Biotechnology for the assistance with SEM. This study was partially supported by the National Council for Scientific and Technological Development $(\mathrm{CNPq})$, the Foundation Coordination for the Improvement Higher Education Personnel (CAPES), Fundação de Apoio a Pesquisa (FAP-DF) and Embrapa Genetic Resources and Biotechnology. Funding was provided by the Air Force Office of Scientific Research (RVL and FT).

1 Vollrath, F. \& Knight, D. P. Liquid crystalline spinning of spider silk. Nature 410, 541-548 (2001).

2 Humenik, M., Smith, A. M. \& Scheibel, T. Recombinant spider silks-biopolymers with potential for future applications. Polymers 3, 640-661 (2011).

3 Brown, C. P., Rosei, F., Traversa, E. \& Licoccia, S. Spider silk as a load bearing biomaterial: tailoring mechanical properties via structural modifications. Nanoscale $\mathbf{3}$, 870-876 (2011).

4 Colgin, M. A. \& Lewis., R. V. Spider minor ampullate silk proteins contain new repetitive sequences and highly conserved non-silk-like 'spacer regions'. Prot. Sci. 7, 667-672 (1998).

5 Gosline, J. M., Guerette, P. A., Ortlepp, C. S. \& Savage, K. N. The mechanical design of spider silks: from fibroin sequence to mechanical function. J. Exp. Biol. 202, 3295-3303 (1999).

6 Guerette, P., Ginzinger, D., Weber, B. \& Gosline, J. Silk properties determined by glandspecific expression of a spider fibroin gene family. Science 272, 112-115 (1996).

7 Hayashi, C. Y. \& Lewis, R. V. Evidence from flagelliform silk cDNA for the structural basis of elasticity and modular nature of spider silks. J. Mol. Biol. 275, 773-784 (1998).

8 Becker, N., Oroudjev, E., Mutz, S., Cleveland, J. P., Hansma, P. K., Hayashi, C. Y., Makarov, D. E. \& Hansma, H. G. Molecular nanosprings in spider capture-silk threads. Nat. Mat. 2, 278-283 (2003).
9 Keten, S. \& Buehler, M. J. Nanostructure and molecular mechanics of spider dragline silk protein assemblies. J. R. Soc. Interface 7, 1709-1721 (2010).

10 Sponner, A., Vater, W., Rommerskirch, W., Vollrath, F., Unger, E., Grosse, F. \& Weisshart, K. The conserved C-termini contribute to the properties of spider silk fibroins. Biochem. Biophys. Res. Commun. 338, 897-902 (2005).

11 Ittah, S., Cohen, S., Garty, S., Cohn, D. \& Gat, U. An essential role for the C-terminal domain of a dragline spider silk protein in directing fiber formation. Biomacromolecules 7, 1790-1795 (2006).

12 Rising, A., Hjalm, G., Engstrom, W. \& Johansson, J. N-terminal nonrepetitive domain common to dragline, flagelliform, and cylindriform spider silk proteins. Biomacromolecules 7, 3120-3124 (2006).

13 Garb, J. E., Ayoub, N. A. \& Hayashi, C. Y. Untangling spider silk evolution with spidroin terminal domains. BMC Evol. Biol. 10, 243 (2010).

14 Hagn, F., Eisoldt, L., Hardy, J. G., Vendrely, C., Coles, M., Scheibel, T. \& Kessler, H. A conserved spider silk domain acts as a molecular switch that controls fibre assembly. Nature 465, 239-242 (2010).

$15 \mathrm{Li}, \mathrm{S}$. F. Y., McGhie, A. J. \& Tang, S. L. Comparative study of the internal structures of kevlar and spider silk by atomic force microscopy. J. Vac. Sci. Tech. A: Vac. Surf. Films 12, 1891 (1994).

16 Vehoff, T., Glišović, A., Schollmeyer, H., Zippelius, A. \& Salditt, T. Mechanical properties of spider dragline silk: humidity, hysteresis, and relaxation. Biophys. J. 293, 4425-4432 (2007).

17 Opell, B. D. \& Bond, J. E. Capture thread extensibility of orb-weaving spiders: testing punctuated and associative explanations of character evolution. Biol. J. Linn. Soc. 70, 107-120 (2000).

18 Köhler, T. \& Volrath, F. Thread biomechanics in the two orb-weaving spiders Araneus diadematus (Araneae, Araneidae) and Uloborus walckenaerius (Araneae, Uloboridae). J. Exp. Biol. 271, 1-17 (1995).

19 Vollrath, F., Barth, P., Basedow, A., Engström, W. \& List, H. Local tolerance to spider silks and protein polymers in vivo. In Vivo 16, 229-234 (2002).

20 Altman, G. H., Diaz, F., Jakuba, C., Calabro, T., Horan, R. L., Chen, J. S., Lu, H., Richmond, J. \& Kaplan, D. L. Silk-based biomaterials. Biomaterials 24, 401-416 (2003).

21 Meinel, L., Hofmann, S., Karageorgiou, V., Kirker-Head, C., McCool, J., Gronowicz, G., Zichner, L., Langer, R., Vunjak-Novakovic, G. \& Kaplan, D. L. The inflammatory responses to silk films in vitro and in vivo. Biomaterials 26, 147-155 (2005).

22 Gellynck, K., Verdonk, P., Forsyth, R., Almqvist, K. F., Van Nimmen, E., Gheysens, T., Mertens, J., Van Langenhove, L., Kiekens, P. \& Verbruggen, G. Biocompatibility and biodegradability of spider egg sac silk. J. Matter. Sci. Mater. Med. 19, 2963-2970 (2008).

23 Teulé, F., Furin, W. A., Cooper, A. R., Duncan, J. R. \& Lewis, R. V. Modifications of spider silk sequences in an attempt to control the mechanical properties of the synthetic fibers. J. Mater. Sci. 42, 8974-8985 (2007).

24 Fahnestock, S. R., Yao, Z. \& Bedzyk, L. A. Microbial production of spider silk proteins. J. Biotechnol. 74, 105-119 (2000).

25 Holland, C., Terry, A. E., Porter, D. \& Vollrath, F. Comparing the rheology of native spider and silkworm spinning dope. Nat. Mat. 5, 870-874 (2006).

26 Teulé, F., Cooper, A. R., Furin, W. A., Bittencourt, D., Rech, E. L., Brooks, A. \& Lewis, R. V. A protocol for the production of recombinant spider silk-like proteins for artificial fiber spinning. Nat. Prot. 4, 341-355 (2009).

27 Knight, D. P. \& Vollrath, F. Changes in element composition along the spinning duct in a Nephila spider. Naturwissenschaften 88, 179-182 (2001).

28 Chen, X., Shao, Z. Z. \& Vollrath, F. The spinning processes for spider silk. Soft Matter 2, 448-451 (2006).

29 Shao, Z. Z. \& Vollrath, F. The effect of solvents on the contraction and mechanical properties of spider silk. Polymer 40, 1799-1806 (1999).

30 Dicko, C., Knight, D., Kenney, J. M. \& Vollrath, F. Conformational polymorphism, stability and aggregation in spider dragline silks proteins. Int. J. Biol. Macromol. 36, 215-224 (2005).

31 Lawrence, B. D., Wharram, S., Kluge, J. A., Leisk, G. G., Omenetto, F. G., Rosenblatt, M. I. \& Kaplan, D. L. Effect of hydration on silk film material properties. Macromol. Biosci. 10, 393-403 (2010).

32 Metwalli, E., Slotta, U., Darko, C., Roth, S. V., Scheibel, T. \& Papadakis, C. M. Structural changes of thin films from recombinant spider silk proteins upon posttreatment. Appl. Phys. 89, 655-661 (2007).

33 Oroudjev, E., Soares, J., Arcdiacono, S., Thompson, J. B., Fossey, S. A. \& Hansma, H. G. Segmented nanofibers of spider dragline silk: atomic force microscopy and singlemolecule force spectroscopy. Proc. Natl Acad. Sci. USA 99, 6460-6465 (2002).

34 Schäfer, A., Vehoff, T., Glišović, A. \& Salditt, T. Spider silk softening by water uptake: an AFM study. Eur. Biophys. J. 37, 197-204 (2008).

35 Chen, X., Shao, Z. \& Marinkovic, N. S. Conformation transition kinetics of regenerated Bombyx mori silk fibroin membrane monitored by time-resolved FTIR spectroscopy. Biophys. Chem. 89, 25-34 (2001).

36 Arcidiacono, S., Mello, C. M., Butler, M., Welsh, E., Soares, J. W., Allen, A., Ziegler, D., Laue, D. \& Chase, S. Aqueous processing and fiber spinning of recombinant spider silks. Macromolecules 35, 1262-1266 (2002).

37 Sneddon, I. N. The relation between load and penetration in the axisymmetric Boussinesq problem for a punch of arbitrary profile. Int. J. Eng. Sci. 3, 47-57 (1965).

38 Domke, J. \& Radmacher, M. Measuring the elastic properties of thin polymer films with the atomic force microscope. Langmuir 14, 3320-3325 (1998).

39 Bar, G., Thomann, Y., Brandsch, R. \& Cantow, H.-J. Factors affecting the height and phase images in tapping mode atomic force microscopy. Study of phase-separated 
polymer blends of poly(ethene-co-styrene) and poly(2,6-dimethyl-1,4-phenylene oxide). Langmuir 13, 3807-3812 (1997).

40 Doerner, M. F. \& Nix, W. D. A method for interpreting the data from depth-sensing indentation instruments. J. Mater. Res. 1, 601-609 (1986).

41 Tranchida, D., Piccarolo, S., Loos, J. \& Alexeev, A. Accurately evaluating Young's modulus of polymers through nanoindentations: a phenomenological correction factor to the Oliver and Pharr procedure. Appl. Phys. Lett. 89, 95-99 (2006).

42 Shao, Z., Vollrath, F., Sirichaisit, J. \& Young, R. J. Analysis of spider silk in native and supercontracted states using Raman spectroscopy. Polymer 40, 2493-2500 (1999).

43 Liu, Y., Shao, Z. \& Vollrath, F. Relationships between supercontraction and mechanical properties of spider silk. Nat. Mater. 4, 901-905 (2005).

44 Landman, U., Luedtke, W. D., Burnham, N. A. \& Colton, R. J. Atomistic mechanisms and dynamics of adhesion, nanoindentation and fracture. Science 248, 454-461 (1990).

45 Burnham, N. A. \& Colton, R. J. Measuring the nanomechanical properties and surface forces of materials using an atomic force microscope. J. Vac. Sci. Technol. 7, 2906-2913 (1989)

46 Jenkins, J. E., Creager, M. S., Lewis, R. V., Holland, G. P. \& Yarger, J. L. Quantitative correlation between the protein primary sequences and secondary structures in spider dragline silks. Biomacromolecules 11, 192-200 (2010).

47 Madsen, B. \& Vollrath, F. Mechanics and morphology of silk drawn from anesthetized spiders. Naturwissenschaften 87, 148-153 (2000).

48 Swanson, B. O., Anderson, S. P., Digiovine, C., Ross, R. N. \& Dorsey, J. P. The evolution of complex biomaterial performance: the case of spider silk. Integr. Comp. Biol. 49 , 21-31 (2009).
49 Hirota, N., Mizuno, K. \& Goto, Y. Group additive contributions to the alcohol-induced alpha-helix formation of melittin: implication for the mechanism of the alcohol effects on proteins. J. Mol. Biol. 275, 365-378 (1998).

50 Blackledge, T. A. \& Hayashi, C. Y. Unraveling the mechanical properties of composite silk threads spun by cribellate orb-weaving spiders. J. Exp. Biol. 209, 3131-3140 (2006).

$51 \mathrm{Xu}$, Z. \& Buehler, M. J. Mechanical energy transfer and dissipation in fibrous betasheet-rich proteins. Phys. Rev. E. Stat. Nonlin. Soft. Matter. Phys. 81, 061910 (2010).

52 Knight, D. P., Knight, M. M. \& Vollrath, F. Beta transition and stress-induced phase separation in the spinning of spider dragline silk. Int. J. Biol. Macromol. 27, 205-210 (2000).

53 Pérez-Rigueiro, J., Plaza, G. R., Torres, F. G., Hijar, A., Hayashi, C., Perea, G. B. Elices, M. \& Guinea, G. V. Supercontraction of dragline silk spun by lynx spiders (Oxyopidae). Int. J. Biol. Macromol. 46, 555-557 (2010).

54 Gould, S. A., Tran, K. T., Spagna, J. C., Moore, A. M. \& Shulman, J. B. Short and long range order of the morphology of silk from Latrodectus hesperus (Black Widow) as characterized by atomic force microscopy. Int. J. Biol. Macromol. 24, 151-157 (1999).

55 Weisenhorn, A. L., Khorsandi, M., Kasas, S., Gotzos, V. \& Butt, H.-J. Deformation and height anomaly of soft surfaces studied with an AFM. Nanotechnology 4, 106-113 (1993).

56 Zhou, H. \& Zhang, Y. Hierarchical chain model of spider capture silk elasticity. Phys. Rev. Lett. 94, 028104 (2005).

57 Li, G., Zhou, P., Shao, Z., Xie, X., Chen, X., Wang, H., Chunyu, L. \& Yu, T. The natural silk spinning process. A nucleation-dependent aggregation mechanism? Eur. J. Biochem. 268, 6600-6606 (2001). 